\title{
Определение оптимального соотношения между дискретизацией и квантованием для СВЧ АЦП
}

\author{
Г. Алексеев ${ }^{1}$, А. Калёнов ${ }^{2}$, И. Мухин, к. т. н. ${ }^{3}$, В. Репин, к. т. н. ${ }^{4}$
}

УДК 621.3 | ВАК 05.27 .01

\begin{abstract}
При создании и использовании аналого-цифровых преобразователей, работающих в СВЧ-диапазоне, актуальна задача обеспечения оптимального соотношения между их частотой дискретизации и разрядностью. О некоторых подходах к решению данной проблемы рассказывается в статье.
\end{abstract}

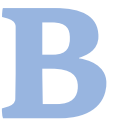

основе преобразования аналогового сигнала в цифровой вид лежит теорема Котельникова связывающая ширину полосы обрабатываемого сигнала с тактовой частотой, выбор которой математически обоснован для любых реальных сигналов. Для низкочастотных приложений аналого-цифровых преобразователей (АЦП), обычно для обработки звукового сигнала, приняты стандартные частоты сэмплирования и разрядности преобразователей. В случае разработки СвЧ-преобразователей возникает проблема увеличения разрядности с повышением частоты дискретизации. Возрастает сложность структуры АЦП, встает вопрос о ее реализуемости в рамках определенных базисных технологий и появляется проблема роста потребляемой мощности

В то же время разработчики аппаратуры выдвигают требования, связанные с ростом скорости передачи потока данных - параметра битрейт, определяемого как произведение разрядности на тактовую частоту, а также требования увеличения динамического диапазона преобразователей. Один из способов расширения динамического диапазона АЦП представлен в [1]. Требование об увеличении скорости потока выдвигается разработчиками зачастую без учета сложностей реализации таких преобразователей и без выбора оптимального соотношения между разрядностью и частотой дискретизации. Очевидно, что частота преобразования должна соответствовать критерию Найквиста для полосы входных частот, но при этом точность, определяемая шагом (уровнями)

МИЭТ, кафедра интегральной электроники и микросистем (ИЭМС), аспирант, g.alekseev@mri-progress.ru.

МИЭТ, кафедра ИЭМС, аспирант, kalenov@mri-progress.ru. АО «НИИМА «Прогресс», начальник отделения,

iimukhin@mri-progress.ru.

АО «НИИМА «Прогресс», заместитель начальника отделения, repin@mri-progress.ru. квантования, может быть недостаточна, а при заданной точности не всегда возможно достичь требуемой тактовой частоты.

Проанализируем результат оцифровки произвольного сигнала с помощью идеальных АЦП без учета дифференциальных и интегральных искажений. На рис. 1 представлены два графика, иллюстрирующие обработку произвольного периодического сигнала с помощью быстрого, но грубого (рис. 1а) АЦП и медленного, но точного (рис. 1б) АЦП. На обоих графиках видна существенная погрешность преобразования. Графики изображают величину входного сигнала в зависимости от времени и значения сигналов, полученные на выходе высокоразрядного ЦАП, стоящего после исследуемых идеализированных АЦП, а погрешность преобразования в данном случае определяется как разность между значениями входного сигнала и сигналов с выхода ЦАП в данные моменты времени.

На рис. 2 представлены графики нормированного линейно нарастающего сигнала с различной скоростью нарастания и результаты их обработки АЦП прямого преобразования, у которых тактовая частота равна частоте дискретизации, наиболее распространенными в области СВЧ. Показаны результаты обработки сигнала и последующего его восстановления преобразователями с различными соотношениями шагов квантования и частот дискретизации. Как видно, при высокой крутизне мы получаем избыточное квантование (разрядность), а при низкой крутизне - избыточную частоту преобразования. Избыточным квантованием мы считаем случай, когда за один период дискретизации сигнал изменяется на несколько квантовых уровней. Аналогично и для случая передискретизации, когда нескольким отсчетам соответствует один квантовый уровень.

Представим входной сигнал в виде треугольных импульсов, что является кусочно-линейной моделью синусоидального сигнала. Для того чтобы изменение 


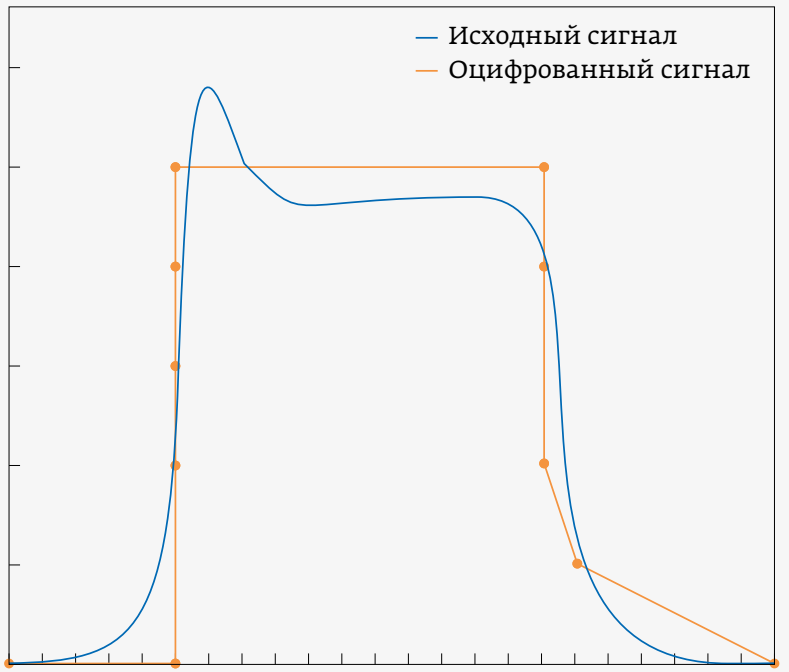

a)

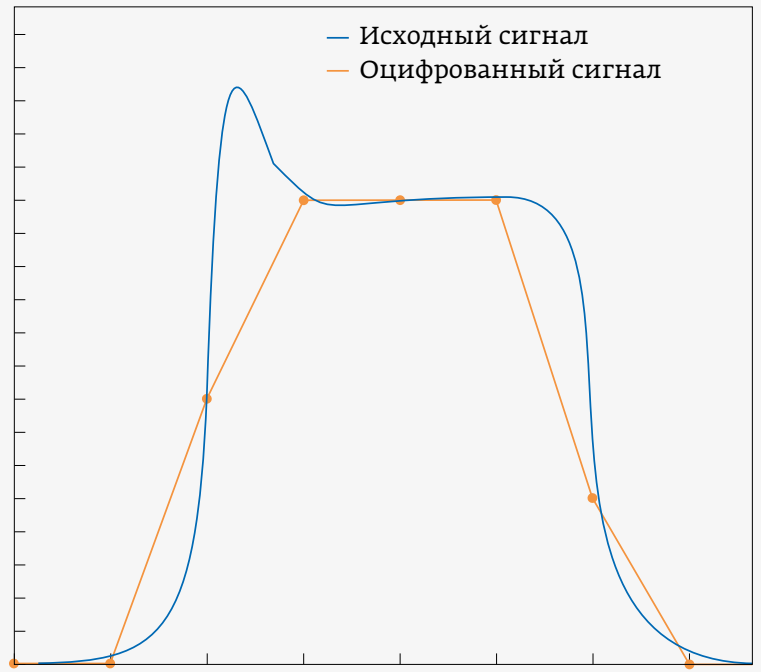

б)

Рис. 1. Обработка сигнала быстрым низкоразрядным АЦП (а) и медленным высокоразрядным АЦП (б)

на один квант происходило за один период дискретизации, скорость нарастания сигнала $V_{\text {н }}$ должна быть равна:

$$
V_{\mathrm{H}}=\frac{\mathrm{U}_{\max }}{2^{\mathrm{N}}} \frac{1}{\mathrm{~T}_{\mathrm{S}}}=\frac{\mathrm{U}_{\max }}{2^{\mathrm{N}}} f_{\mathrm{s}}=u f_{\mathrm{s}}
$$

где $U_{\max }$ - амплитуда входного однополярного сигна-

ла,

$f_{s}$ - тактовая частота,

$\mathrm{T}_{\mathrm{s}}=1 / \mathrm{f}_{\mathrm{s}}$ - период дискретизации,

и - квант преобразования по входному напряже-

нию,

$\mathrm{N}$ - разрядность преобразователя.

Для синусоидального сигнала $U(t)=U_{\max } \cdot \sin (2 \pi f t)$, где $f$ - частота сигнала, а $U_{\max }=u 2^{\mathrm{N}} / 2$, максимальная скорость изменения сигнала $V_{\text {н }}$ получается вычислением производной от $U(t)$, при максимальном значении $\cos (2 \pi f t)=1$. Приравняв скорость нарастания к изменению сигнала на один квант за один период дискретизации получим $V_{\mathrm{H}}=u\left(2^{\mathrm{N}} / 2\right) 2 \pi f=u / \mathrm{T}_{s^{\prime}}$ откуда

$$
f=\frac{f_{s}}{2^{N} \pi} \text {. }
$$

Таким образом, для получения максимальной точности преобразования при линейной аппроксимации сигнала частота дискретизации или разрядность выбираются исходя из заданной скорости нарастания сигнала в соответствии с выражением (1). В случае синусоидального сигнала надо определить максимальную частоту из полосы обрабатываемого сигнала после антиалайзингового фильтра из разложения в ряд
Фурье, задать один из параметров (частоту дискретизации или разрядность) и выбрать второй параметр в соответствии с выражением (2), при этом мы получаем значения частоты дискретизации значительно выше, чем требует теорема Котельникова. В общем случае эффект передискретизации находит широкое применение при обработке сигналов. Если реализация антиалайзингового фильтра с требуемыми значениями частот и величины подавления вызывает трудности, то следует допустить определенную степень

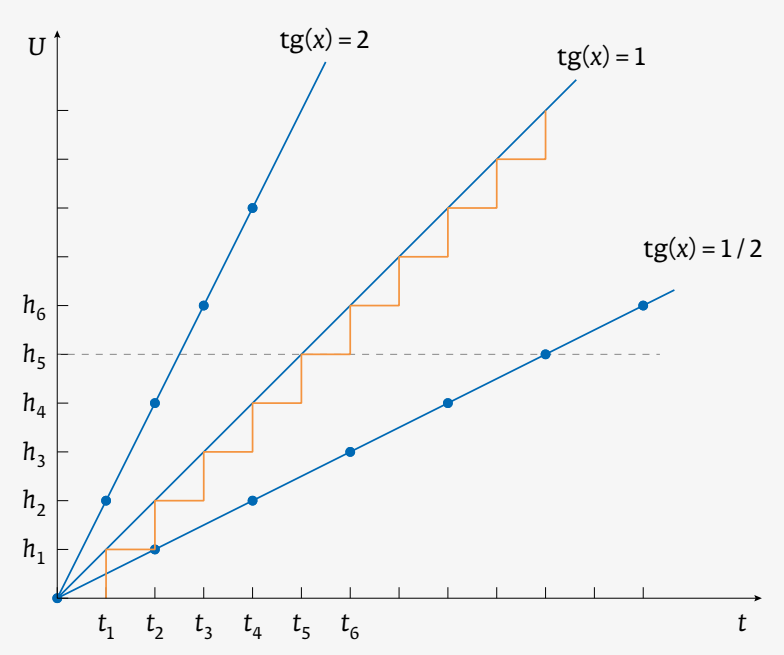

Рис. 2. Реакция на линейный сигнал с различной скоростью нарастания 


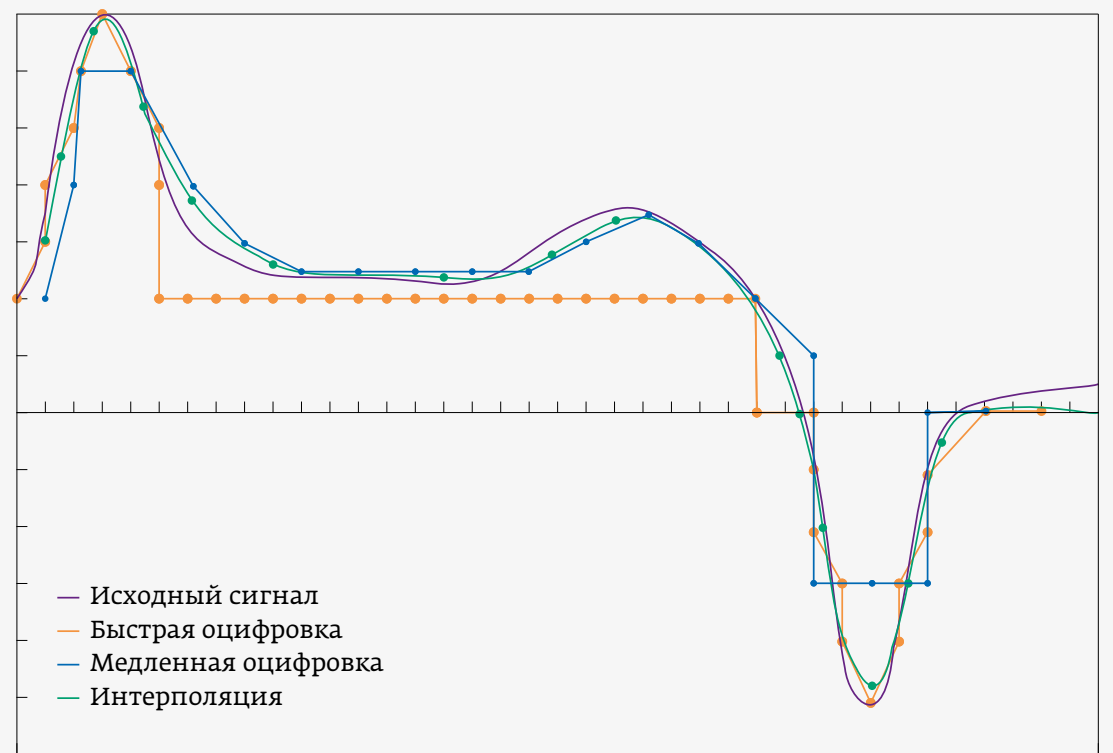

Рис. 3. Обработка сигнала быстрым и медленным АЦП и с учетом интерполяции

передискретизации, выбрав АЦП с более высокой частотой преобразования [2]. С другой стороны, если для потребителя важнейшим параметром является шум квантования или отношение сигнал-шум (SNR), то целесообразно увеличение разрядности. При этом:

$$
\operatorname{SNR}=6,02 \mathrm{~N}+1,76+10 \lg \left(f_{s} /(2 \cdot \mathrm{BW})\right),
$$

где BW - полоса частот оцифровываемого сигнала [2]. Как видно, в реальной практике возникают отклонения от оптимального использования аппаратных возможностей конкретного преобразователя, связанные с дополнительными требованиями по шумам, искажениям и другим параметрам АЦП.

В настоящее время большинство СВЧ АЦП выполняется по структурной схеме, содержащей несколько

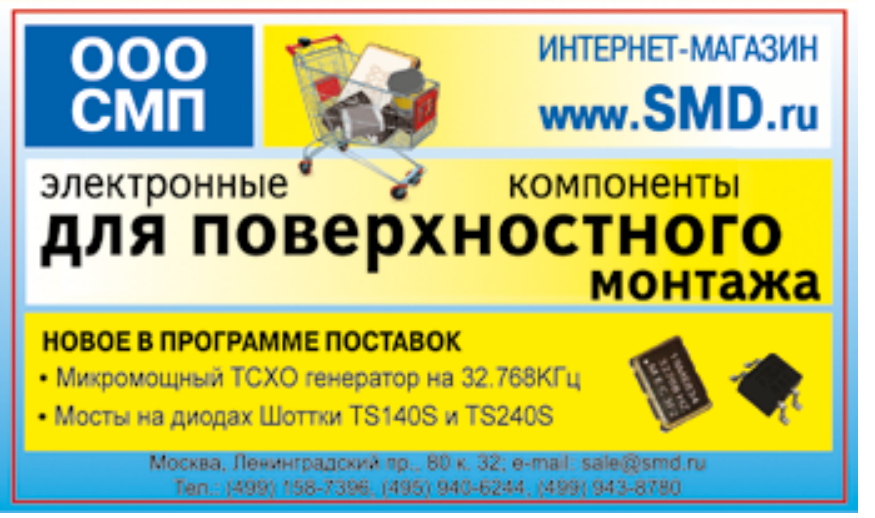

преобразователей, на которые через устройства выборки и хранения (УВХ) подается сигнал с заданным фазовым сдвигом, что позволяет увеличить частоту дискретизации в количество раз, соответствующее числу преобразователей.

Возможно также применение структурной схемы, содержащей несколько преобразователей, которые будут различаться как частотой дискретизации, так и разрядностью. Рассмотрим данный принцип на примере использования двух АЦП: быстрого - с разрядностью $\mathrm{N}_{f}$ и частотой дискретизации $\mathrm{F}_{f}$ и медленного - с разрядностью $\mathrm{N}_{s}$ и частотой дискретизации $F_{s}$ (рис. 3). При этом $\mathrm{N}_{s}=\mathrm{N}_{f}+1$, a $F_{f}=2 F_{s}$. Реализация таких преобразователей проще, чем создание АЦП с разрядностью $\mathrm{N}_{s}$ и частотой дискретизации $\mathrm{F}_{f}$.

При обработке сигнала в преобразователях данного типа учитываются значения, полученные в точках дискретизации медленного АЦП и зафиксированные в его УВХ; а значения, полученные в соседних с ними точках дискретизации быстрого АЦП и сохраненные в его УВХ, корректируются путем интерполяции между данными от быстрого и медленного АЦП. Как видно из рис. 3, точность воспроизведения сигнала с помощью двух АЦП при интерполировании значительно превышает точность обработки отдельными АЦП.

Возможно использование различных структур и элементных базисов для медленного и быстрого АЦП, что позволит снизить потребление энергии при заданной точности преобразования.

Применение АЦП с высокой тактовой частотой увеличивает точность на фронтах сигнала, а АЦП с низкой тактовой частотой, но большей разрядностью, позволит повысить точность преобразования для медленных сигналов, а также снизить зависимость от погрешностей, связанных с джиттером, присущих СВЧ АЦП.

\section{ЛИТЕРАТУРА}

1. Алексеев Г., Морозов Д., Мухин И., Репин В. Расширение динамического диапазона СВЧ АЦП с помощью логарифмических усилителей // ЭЛЕКТРОНИКА: Наука, Технология, Бизнес. 2018. № 6. С. 94-98.

2. Аналого-цифровое преобразование/Под ред. У. Кестера. - М.: ТЕХHOСФЕРА, 2007. 1016 C. 


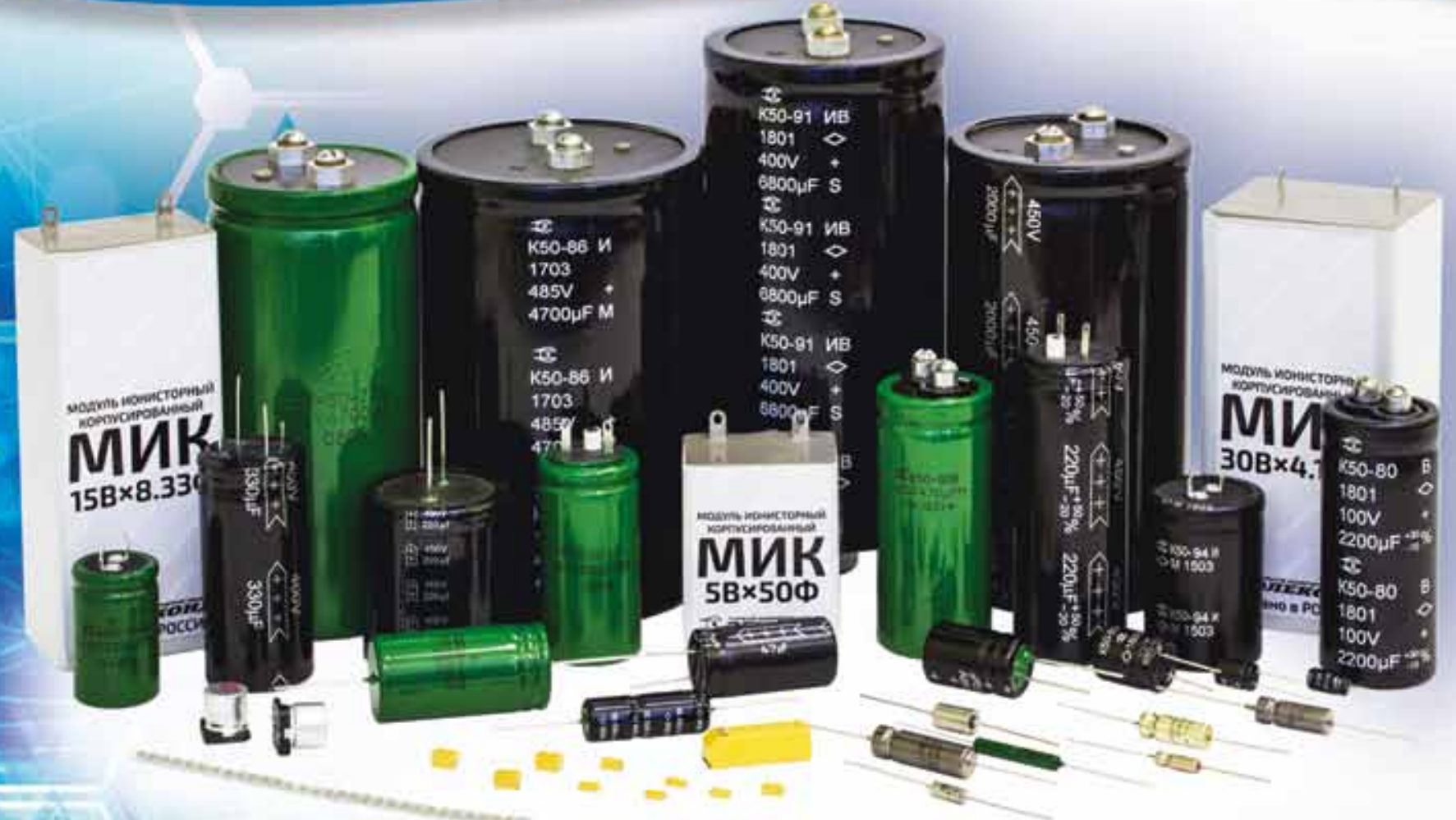

\section{Разработка и производство конденсаторов}

оксидно-электролитические алюминиевые конденсаторы К50-15, К50-17, К50-27, К50-37, К50-68, К50-74, К50-76, К50-77, К50-80, К50-81, К50-83, К50-84, К50-85, К50-86, К50-87, К50-88, К50-89, К50-90, К50-91, К50-92, К50-93, К50-94, К50-95(чип), К50-96, К50-98

объемно-пористые танталовые конденсаторы К52-1, К52-1М, К52-1БМ, К52-1Б, К52-9, К52-11, К52-17, К52-18, К52-19, К52-20, К52-21, К52-24, К52-26(чип), К52-27(чип)

оксидно-полупроводниковые танталовые конденсаторы

К53-1А, К53-7, К53-65(чип), К53-66, К53-68(чип), К53-71(чип), К53-72(чип), К53-74(чип), К53-77(чип), К53-78(чип)

суперконденсаторы (ионисторы) K58-26

накопители электрической энергии на основе модульной сборки суперконденсаторов

Система менеджмента качества сертифицирована на соответствие требованиям ISO 9001

Россия, 427968, Удмуртская Республика, г. Сарапул, ул. Калинина, 3 Тел.: (34147) 2-99-53, 2-99-89, 2-99-77, факс: (34147) 4-32-48, 4-27-53 e-mail: elecond-market@elcudm.ru, http://www.elecond.ru 